\title{
Relaxation patterns of technological residual voltage in the surface layer of high-strength steel when cyclically loaded
}

\author{
Georgi Kravchenko, ${ }^{1, *}$, Konstantin Kravchenko ${ }^{1}$, Andrey Smolyaninov ${ }^{2}$, and Irina \\ Kudryavtseva ${ }^{2}$ \\ ${ }^{1}$ Moscow Aviation Institute (National Technical University), Volokolamckoe shosse, 4, Moscow, \\ 125993, Russia \\ ${ }^{2}$ Voronezh State Technical University, 20 years of October st., 84, Voronezh, 394006, Russia
}

\begin{abstract}
Experimentally investigated patterns of changes in technological residual stresses under the influence of variable pressure in the surface layer became 30XNS2A. A mathematical model of relaxation of residual compressive stresses created by surface plastic deformation techniques with symmetrical cyclical bending of samples has been proposed. An empirical expression is proposed for assessing the final value of residual stresses as a result of cyclic loading, depending on the stress amplitude of a symmetric cycle. An expression is given for estimating the coefficient of relaxation rate of residual compressive stresses from their initial value, amplitude of alternating stresses and material properties. The constants of these expressions are determined for various construction materials. The theoretical dependences describe well the obtained experimental data. To predict the level of residual stress realization under operational loading, a formula was obtained to calculate their change as a result of the action of a step loading block with different amplitudes and duration of their action at each of the stages.
\end{abstract}

\section{Introduction}

Many aircraft parts, such as runway elements, power cylinders of hydro systems, unit nodes and wing mechanization units and many others, are made of high-strength steel, titanium and aluminum alloys, including the widely used steel 30XNS2A. All of these units and nodes in operation are exposed to the force of variable loads, and therefore, their resource is determined by the characteristics of resistance to fatigue and durability.

The methods of improving the quality, reliability and resource of such parts are widely used in the aviation industry methods of improving the quality, reliability and resource of such parts. The main role in increasing the resource of these parts, made of high-strength steels and titanium alloys, is played by residual compressive voltages created in the process of PPD. It is well known that during the operation, under certain external conditions, the amount of residual stresses, and therefore the quality of hardening, can significantly decrease. However, the

\footnotetext{
* Corresponding author: gnkrav@mail.ru
} 
patterns of this decline, the stability and stability of the hardening effect under the influence of variable pressure are not sufficiently understood. The tendency to increase the resource of transport and passenger aircraft to 60-70 thousand. the flight clock makes the problem of studying the relaxation patterns of favorable fields of residual compressive stresses even more relevant.

The degree and speed of relaxation of residual stresses is mainly determined by the physical and mechanical characteristics of the material, the technology of their manufacture and hardening, the level and nature of the load of the part, its geometry and the level of stress concentration, the external environment and the working temperature of the part. It has been experimentally established [1] that the most intense drop in residual stresses is observed in the range of 3 to $10^{4}$ cycles of pressure. And in low-strength materials this drop is the most significant. Thus, if in high-strength steel after the first 10 cycles of pressure residual voltage strains decrease by $40 \%$, and after 100 cycles - by $50 \%$, in aluminum alloy after 100 cycles they are reduced by $80 \%$ [2-4].

The quantitative patterns of relaxation of residual stresses under the influence of variable loads are necessary to predict resistance to fatigue and durability of hardened parts, optimal design of the processes of their surface hardening, and therefore to ensure the durability of parts during operation.

The purpose of this article was to study patterns and analytical description of the relaxation of technological residual stresses in the surface layer of high-strength structural materials when cyclically loaded [5]. The experimental part of the work was carried out on smooth samples of steel $30 \mathrm{XNS} 2 \mathrm{~A}$, heat-treated to $\sigma_{\mathrm{B}}=1700 \mathrm{MP}$. The surface of the samples was reinforced by the crushing of a fraction of different diameters from 0.5 to $2.0 \mathrm{~mm}$ at impact speeds of 20 to $40 \mathrm{~m} / \mathrm{s}$ [6-8]. This hardening provided the initial value of residual squeezing stresses directly near the surface of the sample up to $800 \ldots 1000 \mathrm{MP}$ and the thickness of the hardened layer of material up to $0.5 \mathrm{~mm}$. The amount of residual voltages was measured on the «Strainflex MSF-JM» X-ray voltage analyzer, in chromoradiation. The cyclical ation of the samples was carried out by a clean bend on a symmetrical cycle on the test machine MUI6000 [9-11]. The residual voltages were measured on the working part of the samples, along the axis, when they were removed from the machine after 1, 10, 100, 1000, 5000 and 10000 cycles of pressure.

\section{Experimental}

Experimental studies have shown that the residual compressive voltages in the surface layer became 30XNS2A, created by fractional processing, under the influence of cyclical load can decrease by up to $80 \%$ of its original value $[12,13]$. First of all, the degree of this reduction is determined by the amplitude of cyclical stresses. With amplitudes of stresses equal to approximately the endurance limit and lower, residual stresses are reduced by no more than $50 \%$. The most intense relaxation of residual stresses occurs in the first $10 \ldots 100$ cycles of heat. Then their reduction is less significant and does not exceed $10 \ldots 15 \%$. Cyclical pressure with voltage amps of less than 400 MPA, almost does not cause relaxation of residual compressive stresses [14]. The results of the experiment on relaxation of residual stresses are shown in Figure 1. 


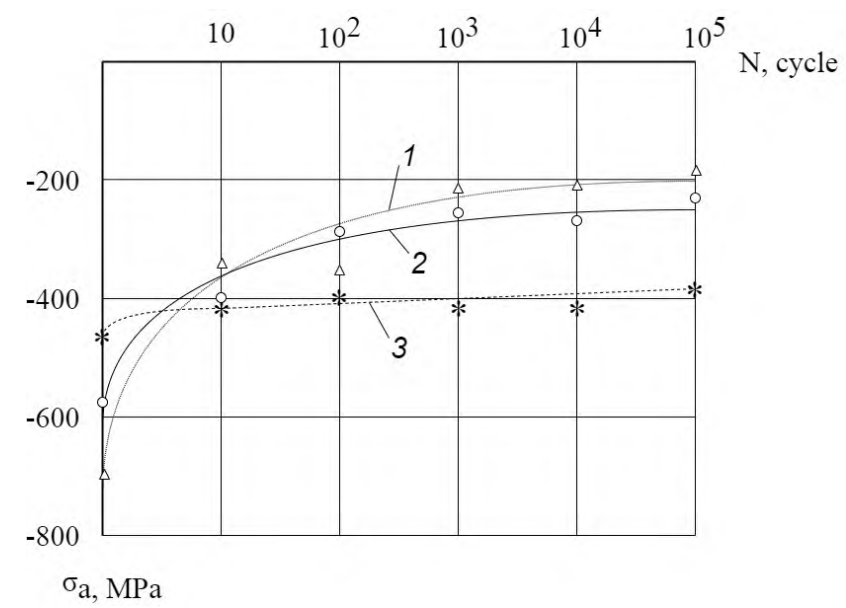

Fig.1. Changes in the magnitude of residual stresses when cyclically loaded with different voltage amplitude values in the surface layer became 30XNS2A $1-\Delta-\sigma_{\mathrm{a}}=1200 \mathrm{MP} ; 2-\mathrm{o}-\sigma_{\mathrm{a}}=800 \mathrm{MP} ; 3$ $\mathrm{x}-\sigma_{\mathrm{a}}=600 \mathrm{MP}$; (points are experimental data; curves are calculated according to the formula (6))

\section{Evaluation}

Based on the results obtained, the following expression is proposed for an analytical description of the dependence of residual stresses on the number of pressure cycles:

$$
\sigma_{o c m}=\frac{2}{\pi} \sigma_{o c m}^{0}\left(\frac{\pi}{2}-\kappa_{v} \operatorname{arctg} N^{v}\right)
$$

where $\sigma_{o c m}^{0}$ - the initial amount of residual stresses after the PPD, N - The number of cycles of the heat-up, $\kappa_{v}$ - relaxation rate, $v$ - the material's relaxation resistance factor.

Analysis of experimental data has substantiated the fact that, at the same amount of amplitude of cyclical stresses, the finite amount of residual squeezing stresses after a sufficiently large number of pressure cycles is independent of their entry level and is determined by the properties of the material, as well as the nature and conditions of its concentration. In addition, there is a threshold value of the voltage amplitude of the external load $\sigma_{a \mu}$, below which the external load does not cause relaxation of residual stresses [15-17]. Based on this provision, an dependence has been obtained to estimate the final value of residual compressive stresses $\sigma_{\text {ocm }}^{\kappa}$ after the number of $\mathrm{N}_{\mathrm{k}}$ cycle cycles:

$$
\begin{aligned}
& \sigma_{o c m}^{\kappa}=\frac{r}{\left(\sigma_{a}-\sigma_{a H}\right)^{s}}, \text { when } \sigma_{a}>\sigma_{a H} \\
& \sigma_{o c m}^{\kappa}=\sigma_{o c m}^{0}, \quad \text { when } \sigma_{a} \leq \sigma_{a H}
\end{aligned}
$$

where is $\sigma_{a}$ - amplitude of cyclical stresses from external load; $\mathrm{r}, \mathrm{S}$ - constants of material.

In the absence of experimental data, you can take $\sigma_{o c m}^{\kappa}=0,4 \ldots 0,6 \sigma_{-1}\left(\sigma_{-1}-\right.$ limited material endurance limit in symmetrical on-loaded cycle);

As a result of the processing of experimental data, constants $r$, $S$ for steel 30XNS2A were obtained $\left(\mathrm{r}=-5,5 * 10^{3}, \mathrm{~S}=0,5\right.$ and $\left.\sigma_{\mathrm{an}}=400 \mathrm{MPa}\right)$ 
Figure 2 provides experimental data on the dependence of the final value of residual squeezing stresses from the amplitude of cyclical stresses for steel 30XNS2A and the estimated curve of this dependence obtained by the formula (2).

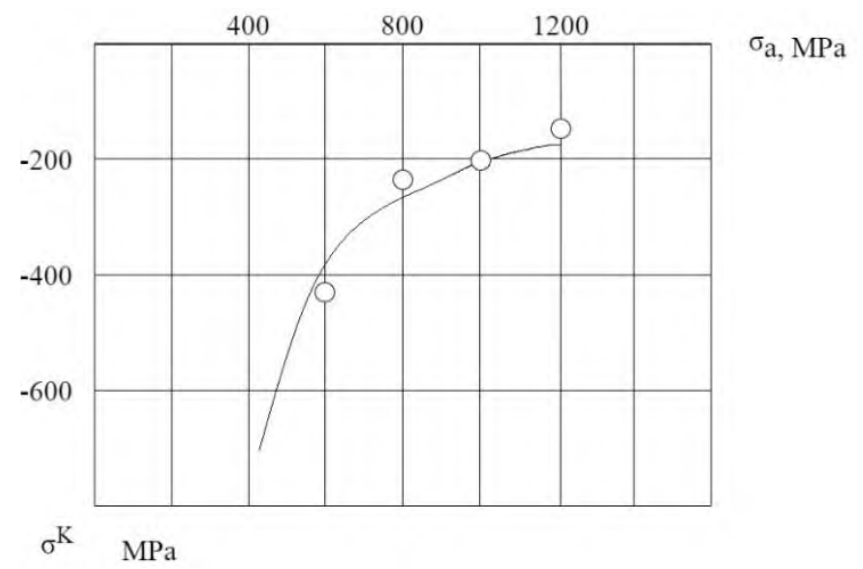

Fig. 2. The dependence of the final value of residual squeezing stresses $\sigma_{\text {ocm }}^{\kappa}$ on the amplitude of cyclical stresses (circles indicate experimental data; the curve is obtained by formula calculation (2) with the number of cycles of the $\mathrm{N}=\mathrm{N \kappa}$ )

The relaxation rate $\kappa_{v}$ in the formula (1) is determined by the amount of cyclical stresses $\sigma_{\mathrm{a}}$ from the external load, the initial value of residual stresses $\sigma_{o c m}^{0}$ and the properties of materials. The coefficient value $\kappa_{v}$ was derived from formula (1) under conditions consistent with a certain number of $\mathrm{N}=\mathrm{N}_{\mathrm{K}}$ cycle cycles, at which the value $\sigma_{o c m}=\sigma_{o c m}^{\kappa}$ :

$$
\begin{aligned}
& \kappa_{v}=c_{N_{\kappa}} \cdot \frac{\sigma_{o c m}^{0}-\sigma_{o c m}^{\kappa}}{\sigma_{o c m}^{0}} o r \\
& \kappa_{v}=c_{N_{\kappa}} \cdot\left(1-c_{p}\right)
\end{aligned}
$$

where is $c_{N_{\kappa}}$ - a ratio corresponding to the boundary number of $\mathrm{N}_{\mathrm{k}}$ cycles, at which $\sigma_{o c m}=\sigma_{o c m}^{\kappa}: c_{N_{\kappa}}=\pi\left(2 \operatorname{arctg} N_{\kappa}^{v}\right)^{-1}, c_{p}=\frac{\sigma_{o c m}^{\kappa}}{\sigma_{o c m}^{0}}$ - degree of relaxation of residual stresses.

With the number of cycles of the loaded $\mathrm{N} \rightarrow \infty$

$$
\kappa_{v}=\frac{\sigma_{o c m}^{0}-\sigma_{o c m}^{\kappa}}{\sigma_{o c m}^{0}}=1-c_{p}
$$

From dependencies (2) and (3) we get:

$$
\begin{aligned}
& \kappa_{v}=c_{N_{\kappa}} \cdot\left(1-\frac{r}{\sigma_{o c m}^{0}\left(\sigma_{a}-\sigma_{a H}\right)^{S}}\right) \text { when } \sigma_{a}>\sigma_{a H} \\
& \kappa_{v}=0, \text { when } \sigma_{a}=\sigma_{a H}
\end{aligned}
$$


Based on the equation (5) on Figure 3, the relaxation rate ratio scan on the amplitude of cyclical stresses and the initial value of residual stresses is built. $-\kappa_{v}=f\left(\sigma_{a}, \sigma_{o c m}^{0}\right)$.

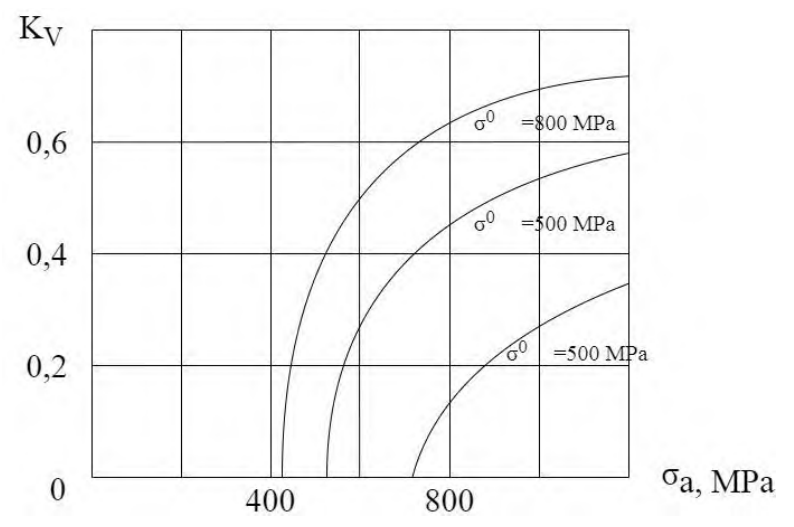

Fig. 3. The dependence of the relaxation rate on the amplitude of cyclical stresses $\sigma_{a}$ for different values of initial residual stresses $\sigma_{o c m}^{0}$

From formulas, (1) and (5) finally get expression to conduct calculations of relaxation of residual compressive stresses in the surface layer of material (when $\sigma_{a}>\sigma_{a H}$ ):

$$
\begin{aligned}
& \sigma_{o c m}=\frac{2}{\pi} \sigma_{o c m}^{0} \times \\
& \times\left(\frac{\pi}{2}-c_{N_{\kappa}}\left[1-\frac{r}{\sigma_{o c m}\left(\sigma_{a}-\sigma_{a H}\right)^{S}}\right] \operatorname{arctg} N^{v}\right)
\end{aligned}
$$

Relaxation curves of residual stresses (for steel 30XNS2A) obtained by formula (6) at $v=0,2$ are applied to Figure 1, from which it is clear that they correspond well to experimental data[18]. Figure 4 shows the estimated curves at the value of $\sigma_{o c m}^{0}=800 \mathrm{MPa}$, illustrating the effect of the amplitude of cyclical stresses on the degree of relaxation of residual compressive stresses.

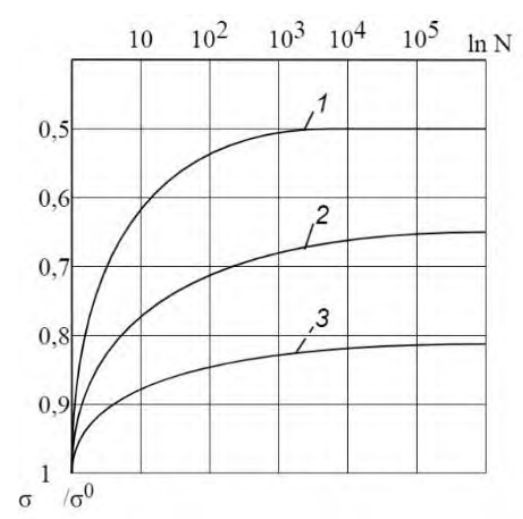

Fig. 4. Estimated relaxation curves of residual stresses in steel $30 \mathrm{XNS} 2 \mathrm{~A}$ at $\sigma_{\mathrm{OCT}}^{\circ}=800 \mathrm{MPa}$ and different voltage amplitude values: $1-\sigma_{\mathrm{a}}=1200 \mathrm{MPA} ; 2-\sigma_{\mathrm{a}}=800 \mathrm{MPA} ; 3-\sigma_{\mathrm{a}}=600 \mathrm{MPA}$

The rate of relaxation of residual stresses can be estimated by formula: 


$$
\begin{aligned}
& V_{\sigma_{\text {ocm }}}=-\sigma_{o c m}^{0} \cdot \frac{2}{\pi} \cdot c_{N_{\kappa}} \times \\
& \times\left[1-\frac{r}{\sigma_{o c m}^{0}\left(\sigma_{a}-\sigma_{a H}\right)^{S}}\right] \cdot \frac{v N^{\nu-1}}{1+N^{2 v}}
\end{aligned}
$$

Based on the experiments and literary data, the parameters of the formula (6) were obtained for some structural materials. Figure. 7 shows the estimated relaxation curves of the residual compressive stresses for steel 30XNS2A $\left(\sigma_{\mathrm{B}}=1700 \mathrm{MPa}, v=0.2\right)$, titanium alloy VTK-1 $\left(\sigma_{\mathrm{B}}=1150 \mathrm{MPa}, v=0.25\right)$ and aluminum alloy D16AT $\left(\sigma_{\mathrm{B}}=430 \mathrm{MPa}, v=0,5\right)$. Curves are obtained at the initial level of residual stresses $\sigma_{o c m}^{0}=0,7 \sigma_{0,2}$ and at the amplitude of cyclical stresses $\sigma_{a}=0,5 \sigma_{0,2}$. From the graph to the Fig. 5 it is seen that with the decrease in the level of strength of the material, relaxation of residual stresses occurs more intensively and by a greater magnitude.

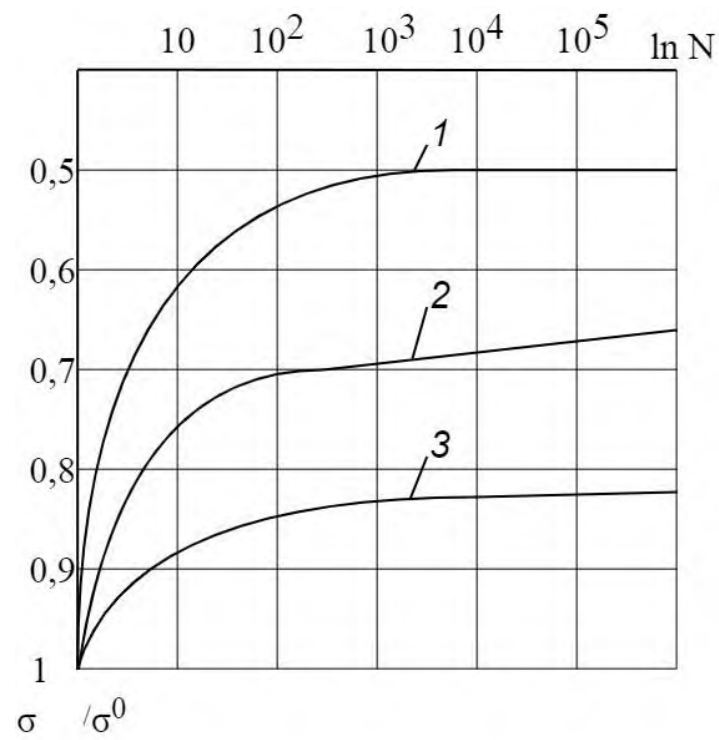

Fig. 5. Estimated relaxation curves of residual stresses when $\sigma_{\text {ocm }}^{0}=0,7 \sigma_{0,2}$ и $\sigma_{\alpha}=0,5 \sigma_{0,2}$ for different materials: 1 - D16AT, 2 - VT3-1, 3 - 30XNS2A

After the $\mathrm{N}=10^{4}$ cycles of pressure, the residual voltages in the D16AT alloy are reduced by $50 \%$, in the alloy of VT3-1 by $33 \%$, and in steel 30 XNS 2 A - only by $17 \%$. This suggests that the use of PPD methods is appropriate; primarily to increase resistance to fatigue of parts from high-strength materials, where technological residual voltage spling is more stable during operation [19-20].

\section{Conclusions}

1. The proposed expression (6) for the relaxation of technological residual stresses in the surface layer of steel 30XNS2A showed sufficient for engineering methods to assess the convergence of the results of calculations with experimental data. 
2. For each material there is a threshold of the amplitude of stresses from the external load of the yuan $\sigma_{\text {an }}$, below which the external cyclical loading does not cause relaxation of the residual compressive stresses created by the methods of PPD.

3. The final amount of the technological residual stresses $\sigma_{\text {ocm }}^{K}$ created by the PPD methods does not depend on their original, initial value and is determined only by the amplitude of stresses and the duration of the external variable load.

\section{References}

1. G. N. Kravchenko, K. G. Kravchenko, I. V. Gerasimov, Recovery of fatigue life of $30 H G S N 2 A$ steel aircraft parts by repeated shot peening, Journal of Physics: Conference Series, 1399(4) (2019)

2. G. N. Kravchenko, K. G. Kravchenko, Restoring the Fatigue Life of Surface-Hardened Airplane Parts by Repeated Shot Hardening. ISSN 1068-798X, Russian Engineering Research, 40 (2020)

3. Y. Deniskin, P. Miroshnichenko, A. Smolyaninov, Geometric modeling of surfaces dependent cross sections in the tasks of spinning and laying, E3S Web of Conferences (2019) DOI: $10.1051 / \mathrm{e} 3$ sconf/201911001057

4. I. Pocebneva, Y. Deniskin, A. Yerokhin, V. Artiukh, V. Vershinin, Simulation of an aerodynamic profile with sections of ad hoc concavity, E3S Web of Conferences, 110, 01074

5. Y. I. Bityukov, Y. I. Deniskin, I. V. Pocebneva, Construction of Smooth Biorthogonal Waves on Triangulated Spaces, Proceedings - 2019 International Russian Automation Conference, RusAutoCon (2019) DOI: 10.1109/RUSAUTOCON.2019.8867785

6. E. Matys, Y. Deniskin, E. Stativa, D. Shlychkov, Special features of obtaining fine powders for additive technologies, E3S Web of Conferences (2019) DOI: 10.1051/e3sconf/201911001057

7. I. M. Artamonov, A. R. Deniskina, Yu. I. Deniskin, Formation of a single information object for targeted solutions in the aerospace industry, Science Industry Defense Proceedings of the XIX All-Russian Scientific and Technical Conference: in 4 volumes. Ed. S.D. Salenko, 57-61 (2018)

8. Y. I. Bityukov, Y. I. Deniskin, Chaikin algorithm and its generalization, 2016 Dynamics of Systems, Mechanisms and Machines, Dynamics, 7818981 (2016)

9. Y. I. Bityukov, Y. I. Deniskin, G. Y. Deniskina, Spline wavelets use for output processes analysis of multi-dimensional non-stationary linear control systems, Journal of Physics: Conference Series, 944(1), 012018

10. A. V. Smolyaninov, I. V. Pocebneva, L. V. Chernenkaya, Mathematical Model of Asynchronous Motor with Frequency-Cascade Regulation, Proceedings 2019 International Russian Automation Conference, RusAutoCon 8867604 (2019)

11. O. Dolgov, S. Bibikov, I. Pocebneva, Elements of the synthesis method for the layout of a front-line aircraft, E3S Web of Conference, 110, 01068 (2019)

12. V. E. Mager, V. E. Belousov, E. N. Desyatirikova, S. A. Ivanov, I. V. Pocebneva, Information processing algorithm at creation of optimum structure of the self-adjusted technical system in quality parameters, Proceedings of 2017 IEEE 2nd International Conference on Control in Technical Systems, CTS, 8109503, 114-117 (2017) 
13. E. N. Desyatirikova, L. V. Chernenkaya, V. E. Mager, Subsystem for On-line Diagnostics of Cutting Process in Flexible Manufacturing, Proceedings - 2019 International Russian Automation Conference, RusAutoCon, 8867814 (2019)

14. E. N. Desyatirikova, L. V. Chernenkaya, V. E. Mager, Method of calculating a priori reliability of elements of complex system, International Russian Automation Conference, RusAutoCon 8501747 (2018)

15. G. N. Kravchenko, Yu. I. Popov, K. G. Kravchenko, Probability prediction of the appearance of overload cycles of variable load case in parts of the aircraft chassis, IOP Conference Series: Materials Science and Engineering, 734(1), 012014

16. E. Protopopov, S. Dobrykh, Y. Trofimova, P. Malenko, A. Valter, A. Protopopov, Reflection of strengthening results in values of generalized degrees of metallicity and covalence is principle to new strategy of designing alloys, Scientific Reports, 10(1), 2050 (2020)

17. A. Dehghani, F. Aslani, The synergistic effects of shape memory alloy, steel, and carbon fibres with polyvinyl alcohol fibres in hybrid strain-hardening cementitious composites, Construction and Building Materials, 252, 119061 (2020)

18. S. S. M. Tavares, R. T. Batista, R. V. Landim, J. A. C. Velasco, L. F. Senna, Investigation of the effect of low temperature aging on the mechanical properties and susceptibility to sulfide stress corrosion cracking of $22 \% \mathrm{Cr}$ duplex stainless steel, Engineering Failure Analysis, 113,104553 (2020)

19. M. Yakhchalian, N. Asgarkhani, M. Yakhchalian, Evaluation of deflection amplification factor for steel buckling restrained braced frames, Journal of Building Engineering, 30, 101228 (2020)

20. J. Chen, H. Liu, T.-M. Chan, Material properties and residual stresses of cold-formed octagonal hollow sections, Journal of Constructional Steel Research, 170, 106078 (2020) 